\title{
Quantum chromodynamics: Working group report
}

\author{
Coordinator: K SRIDHAR ${ }^{1}$
}

Working Group Members: Sunanda Banerjee ${ }^{1}$, Swagato Banerjee ${ }^{1}$, Rahul Basu ${ }^{5}$, Fawzi Boudjema ${ }^{6}$, Michel Fontannaz ${ }^{7}$, Rajiv Gavai ${ }^{1}, \mathrm{~J} \mathrm{Gayler}^{8}$, Rohini Godbole ${ }^{2}$, Sourendu Gupta ${ }^{1}$, D Indumathi ${ }^{3}$, Michael Krämer ${ }^{4}$, Prakash Mathews ${ }^{1}$, Hiranmaya Mishra ${ }^{9}$, P Poulose ${ }^{1}$, Probir Roy ${ }^{1}, K_{\text {Sridhar }}{ }^{1}$

${ }^{1}$ Tata Institute of Fundamental Research, Homi Bhabha Road, Mumbai 400005 , India

${ }^{2}$ Centre for Theoretical Studies, Indian Institute of Science, Bangalore 560012, India

${ }^{3}$ Mehta Research Institute, Chhatnag Road, Jhusi, Allahabad 221 506, India

${ }^{4}$ Rutherford Appleton Lab., Chilton, Didcot, Oxfordshire, OX11 0QX, England

${ }^{5}$ The Institute of Mathematical Sciences, C.I.T. Campus, Taramani, Chennai 600113 , India

${ }^{6}$ LAPP-CNRS, ENSLAPP, Chemin de Bellevue, F-74941 Annecy-la-Vienx, France

${ }^{7}$ Laboratoire de Physique Theorique el Hantes Energie, Universite Paris 11, Batiment 210, F-91405 Orsay Cedex, France

${ }^{8}$ DESY, Notkestr. 85, D-22603 Hamburg, Germany

${ }^{9}$ Theory Division, Physical Research Laboratory, Navrangpura, Ahmedabad 380009 , India

Keywords. Quantum chromodynamics; structure functions; quarkonium production.

PACS No. 12.38

\section{Introduction}

In the QCD working group, several topics were identified and, in this report, the problems which were chosen for investigation will be outlined and the status of the investigations will be presented.

The different topics that were identified are as follows:

- Quarkonia

- Photons

- Low- $x$ physics

- Spin physics

- Glueballs

\section{Quarkonia}

In the last few years, there has been a tremendous advance in the understanding of the production of quarkonia at high-energy colliders. In particular, $J / \psi$ production has been studied in detail both experimentally and theoretically. The first experimental results on $J / \psi$ production at large- $p_{T}$ at the Tevatron showed a huge excess as 


\section{K Sridhar}

compared to the predictions of existing models of quarkonium production. Since then it has been shown that the older models of quarkonium production (like the colour-singlet model) were incomplete because they neglected the colour-octet components of the quarkonium wave-function predicted by the effective field theory approach called non-relativistic QCD (NRQCD). By including the colour-octet components, the discrepancy between theory and the Tevatron experiment can be resolved [1].

In NRQCD, the cross-section for the production of a quarkonium resonance admits of a factorization into a short-distance part which can be computed perturbatively and a non-perturbative part. The non-perturbative part has a rigorous definition in terms of the operators of NRQCD, and while it is, in principle, possible to use lattice techniques to evaluate them, our present knowledge of these are from the fits to the data from Tevatron. Other tests and consistency checks are necessary to genuinely test the NRQCD approach. With this in mind two different problems have been identified in this working group.

- Quarkonium production at the LHC (M Krämer and K Sridhar): It is proposed to study the production of charmonium resonances at the large hadron collider (LHC). These include $J / \psi, \psi^{\prime}$ and $\chi_{c}$ production at the LHC. A study of bottomonium resonances is also planned. Other channels like associated $J / \psi+\gamma$ production will also be looked into. At large values of $p_{T}$ which are likely to be accessible at the LHC, the fragmentation picture will be almost exact and this study could be used to test the fragmentation picture. Also at low- $p_{T}$ values, interesting low- $x$ effects may be studied.

- $\chi_{c}$ feed-down effects to $J / \psi$ polarization (M Krämer and $S$ Gupta): A very interesting test of the NRQCD predictions come from polarization studies. This is because at large $p_{T}$ the theory predicts a very large transverse polarization of the produced $J / \psi$. This is expected to decrease with decreasing $p_{T}$ and the predictions have been obtained for the whole range of $p_{T}$ values relevant to the Tevatron experiment [2]. However, these predictions have been made for the case of direct $J / \psi$ production without taking into account the $J / \psi$ 's that could come from the decays of the $\chi_{c}$ 's. Experimentally, while it is possible to separate the $\chi_{c}$ contribution by detecting the associated soft photons, it is not very easy to do so. The predictions for $J / \psi$ polarization which include the effects of the decays of the $\chi_{c}$ 's will, therefore, be a very useful check and serve to test NRQCD.

- $J / \psi+\gamma$ production at the Tevatron ( $R$ Basu, $P$ Mathews and $K$ Sridhar): The associated production of a $J / \psi$ and a photon at large transverse momentum has been suggested as an interesting probe of quarkonium production mechanisms. The signal for this process can be selected by $p_{T}$ balancing. At the Tevatron, a reasonable number of such events are expected and should be observable with the currently available luminosity. This process has been studied within the NRQCD framework at tree level. The effects of higher order QCD diagrams can provide interesting observable effects related to the fragmentation of gluons. $R$ Basu, $\mathrm{P}$ Mathews and $\mathrm{K}$ Sridhar have undertaken a study of these higher-order effects. Much of the analytical part of the project is completed and the numerical work is underway. 


\section{Photons}

In this subtopic, several problems were identified. These are as follows:

- $J / \psi$ production via resolved photons at $\gamma-\gamma$ colliders ( $R M$ Godbole, D Indumathi and $M$ Krämer): The proposal is to study the production of $J / \psi$ via single-resolved photons in $\gamma-\gamma$ collisions within the NRQCD approach. The colour-octet channels can give large $(\sim 10)$ enhancements and preliminary estimates indicate event rates of the order of 50 at Tristan. Further, the study of $J / \psi$ production by single-resolved processes at LEP II may also be interesting. (A detailed write-up submitted by the authors is attached at the end of this report).

- $Q C D$ backgrounds to $H \rightarrow b \bar{b}$ at $\gamma-\gamma$ colliders (F Boudjema, $R M$ Godbole and $S$ Gupta): At high-energy $\gamma-\gamma$ colliders, one important programme is expected to be the search for the production of the Higgs. While the production can proceed through the direct channel, a large contribution to the production process is expected via both single- and double-resolved photon channels. The produced Higgs will be identified by its decay into a $b \bar{b}$. It is proposed to calculate the backgrounds coming from conventional QCD subprocesses to the $b \bar{b}$ channel for both the single- and double-resolved photon cases.

- Recombination effects in jet production at $\gamma-\gamma$ colliders ( $M$ Fontannaz, $R M$ Godbole, $S$ Gupta and $R$ Basu): In small- $p_{T}$ jet production, saturation or recombination effects could lead to a suppression of the minijet production cross-section. This has been studied at $p-p$ colliders and it is proposed to use these studies to see if similar effects in $\gamma-\gamma$ colliders could be studied.

\section{Low-x}

One of the outstanding problems in low- $x$ QCD is to use the data from HERA to distinguish between various resummation schemes like DGLAP, BFKL etc. At present the data from HERA on the structure functon $F_{2}$ is insufficient to distinguish between these various schemes. This is due to $k_{T}$ diffusion in BFKL which allows $k_{T}$ to diffuse into a region of very low values where perturbation theory no longer holds. This is in contrast to DGLAP where $k_{T}$ ordering constrains it to lie in a region far removed from the infrared.

The solution is to study special final state configurations where diffusion into the IR region is minimized by fixing the start and end point of evolution for the above region. This is the idea behind the suggestion by Mueller on measuring an observable that is less inclusive than $F_{2}$ and he suggested studying DIS events at small $x$ containing an identified jet emitted close to the jet of proton fragments. Such events have now been studied and seem to show a preference for BFKL evolution [3]. However the situation is still far from clear. There are few theoretical calculations in resummed DGLAP. Most predictions for the final state observables are derived from Monte Carlo models whose drawback is their complexity and flexibility to model hadronization. This makes it difficult to pin down which feature of the theoretical input is being tested when compared with the data for the various Monte Carlos in the market like MEPJET, HERWIG, LEPTO etc. On the other hand no Monte Carlo exists yet for BFKL evolution though some attempts have been made in that direction by ARIADNE. 
- Single particle production at large p $p_{T}$ using DGLAP (R Basu, $R$ V Gavai and $R M$ Godbole): Many of the ambiguities inherent in the forward jet event calculations mentioned above can be overcome by considering single particle emission at relatively large $p_{T}$ in the central region. Such an event is more immune to hadronization effects and more directly reflects $\ln k_{T}^{2}$ diffusion from the BFKL ladder. The theoretical calculation has been done in [4] and preliminary data is shown in figure 5 of this paper. Moreover once other effects are taken into account like resolved photon processes it is likely that ordinary DGLAP dynamics can explain the diagram. Recently there has been some study of resolved photon processes in this context [5]. R Basu, R V Gavai and $\mathrm{R} M$ Godbole are now in the process of doing the calculation of single particle emission at large $p_{T}$ in the context of DGLAP dynamics. This would provide an unambiguous comparison for the two approaches. This involves modifying the MEPJET program which calculates jets in DIS both with and without a forward jet. The fragmentation formulae in the MEPJET program have to be replaced by suitable fragmentation functions for partons going into pions and kaons rather than jets and the program modified accordingly to take care of infrared cancellations. The last is required as the infrared singularities that get absorbed in the former case in the definition of structure functions are different from those which get absorbed in the definition of the fragmentation function in the latter.

- Double asymptotic scaling in the Drell-Yan process ( $R$ Basu and S Gupta): Doubleasymptotic scaling (DAS) of structure functions has been seen by the HERA experiments for large $\log (1 / x)$ and $\log \left(Q^{2} / \Lambda^{2}\right)$. This implies a similar scaling of other hard QCD cross sections. For example, in a Drell-Yan experiment at large centre of mass energy, $\sqrt{S}$, at fixed rapidity, $y \simeq 0$, when the pair mass, $M$, is such that both $\log \left(S / M^{2}\right)$ and $\log \left(M^{2} / \Lambda^{2}\right)$ are large, the DAS approximation may be expected to hold good. $\mathrm{R}$ Basu and S Gupta plan to look at DAS and corrections in such hard QCD processes.

\section{Spin physics}

In the years following the observation of the so-called spin crisis [6], more data from SMC and HERMES have become available. More reliable parametrizations of polarized parton distributions at LO and NLO have now become available [7]. Nevertheless, our knowledge of the polarized sea-quark density and the polarized gluon density in the proton is limited and more information on these quantities will provide inputs for more reliable parton distribution parametrizations.

- Charged meson production in polarized p - p collisions (S Gupta and K Sridhar): One way of getting a handle on the sea quark content of a polarized proton is to study charged meson production in polarized $p-p$ collisions. It is proposed to study the production of $\pi^{ \pm}, K^{ \pm}$in polarized $p-p$ collisions at RHIC or HERA. These processes will directly constrain the non-strange and the strange sea in the polarized proton.

- Measuring $\Delta g$ in polarized p - p collisions ( $S$ Gupta, D Indumathi and P Mathews): There have been several proposals on strategies to measure the gluon density in a polarized proton. Direct photon production, jet production and large- $p_{T}$ Drell-Yan in polarized $p-p$ collisions have been suggested in this regard. S Gupta, D Indumathi and $\mathrm{P}$ Mathews propose to review the status of these studies and attempt a more 
complete study of these processes including the effects of higher order. The study of higher order effects will allow for a more precise determination of the polarized gluon density.

\section{Glueballs}

In recent years, there have been several isosinglet meson candidates for glueballs but there is no clear-cut evidence for the existence of a glueball state. It is, therefore, interesting to look for the existence of glueballs in high-energy colliders.

- Gluon jet fragmentation to glueballs ( $S$ Banerjee, $P$ Roy and $K$ Sridhar): One possible process for the production of glueballs at high-energy colliders is via the fragmentation of an energetic gluon jet into a glueball state. A sum rule to constrain the fragmentation function has been proposed in ref. [8]. The idea is to use this input and make a simple parametrization of the gluon $\rightarrow$ glueball fragmentation function. With this input, the plan is to look at the prospects of glueball searches at LEP II. At LEP II the number of reconstructed gluon jets (by energy tagging, for example) is large. For a $20 \%$ probability for a gluon to fragment into a glueball state and assuming a $10 \%$ branching of the glueball into a $K_{S}^{0} K_{S}^{0}$ final state it is estimated that one would be left with about a few hundred candidate events. A more precise estimate including the effect of the $Q^{2}$ evolution of the fragmentation function is being attempted.

\section{References}

[1] See the review by $M$ Krämer, Quarkonium Production in this volume

[2] M Beneke and M Krämer, Phys. Rev. D55, 5269 (1997)

[3] See the review by R Basu, Low $x$ and Structure Functions in this volume

[4] J Kwiecinski, S C Lang and A D Martin, preprint DTP-97-56, hep-ph/9707240

[5] H Jung, L Jonsson and H Kuster, preprint DESY-98-051, hep-ph/9805396

[6] For a review see, M Anselmino, A Efremov and E Leader, Phys. Rep. 261, 1 (1995)

[7] W Vogelsang, Acta Phys. Polon. B29, 1189 (1998)

[8] P Roy and T F Walsh, Phys. Lett. B78, 62 (1978)

\section{$J / \psi$ production through resolved photon processes at $e^{+} e^{-}$colliders}

\section{R M Godbole, D Indumathi and M Krämer}

There has been considerable interest in the production of $J / \psi$ at various colliders ever since the large discrepancy between the measured rate of $J / \psi$ production and the (much smaller) prediction of the colour singlet (CS) model was first observed at the Tevatron [1]. An analysis of the data [2] using the NRQCD factorization approach by Bodwin, Braaten and Lepage [3] yielded colour octet (CO) contributions which seemed almost an order of magnitude larger than the CS term. However, later data from HERA [4] did not see the anticipated excess [5], especially at large $z$ values (where $z$ is the inelasticity variable); this may be attributed to the larger uncertainty of the nonperturbative colour octet matrix 


\section{K Sridhar}

elements $\left\langle 0\left|\mathcal{O}_{n}^{J / \psi}\right| 0\right\rangle$, that contribute in the large- $z$ region. It is therefore interesting to estimate the $\mathrm{CO}$ as well as $\mathrm{CS}$ contributions to $J / \psi$ production in various other processes.

Here, we examine the dependence of $\mathrm{CO} \mathrm{J} / \psi$ photoproduction on the various NRQCD matrix elements in $e^{+} e^{-}$colliders. Apart from the direct contribution, there are contributions from diagrams where either one or both of the photons is resolved, so that the underlying parton structure is probed. We are concerned here with these resolved photon contributions, the direct contribution being small, as has already been observed for the case of $\gamma \gamma$ colliders [6].

In particular, there are both colour singlet (CS) and colour octet (CO) contributions to each of these processes. The CS cross section is well known [7]; in fact, it has long since been established that the once resolved (1-res) photon contribution dominates the twice resolved (2-res) photon contribution in the CS case; this was in fact used to estimate the gluon content of the photon [8].

However, the matrix elements in the CO case, with $n=\left[{ }^{3} S_{1}, 8\right],\left[{ }^{1} S_{0}, 8\right]$, and $\left[{ }^{3} P_{J}, 8\right]$, $J=0,1,2$, are not as well established and have recently been obtained from $J / \psi$ production at the Tevatron [2]. Though these are estimated to be about two orders of magnitude smaller than the CS matrix element, the CO contributions are not expected to be small since they correspond to diagrams of lower order in the strong coupling $\alpha_{s}$, or are enhanced by $t$-channel gluon exchange, forbidden in the leading-order colour singlet cross section.

We therefore use the estimated CS and CO matrix elements to compute the $J / \psi$ production cross section in $e^{+} e^{-}$collisions. One of our main results is that the inclusion of $\mathrm{CO}$ terms does not affect the result that the 1-res dominates the 2-res contributions. We find, in general, that the $\mathrm{CO}$ contribution is much larger than the CS one; this may also reflect the fact that we have used octet matrix elements from the Tevatron fits which may overestimate $J / \psi$ production at HERA. However, independently of this, the 1-res contribution dominates. This is in contrast to the ep case, for example, at HERA, where the resolved photon contribution (corresponding to the 2-res term of $\gamma \gamma$ ) is an appreciable fraction of the direct one (corresponding to the 1-res term of $\gamma \gamma$ ) [9]. This is seen in figure 1 where we have plotted the cross section for $J / \psi$ production, $\int_{p_{T, \min }}^{p_{T, \max }}\left(\mathrm{d} \sigma / \mathrm{d} p_{T}\right) \mathrm{d} p_{T}$, where $p_{T, \text { max }}$ is the kinematical limit, $p_{T, \max }=\sqrt{s} / 2$, and we have set $p_{T, \min }=1 \mathrm{GeV}$.

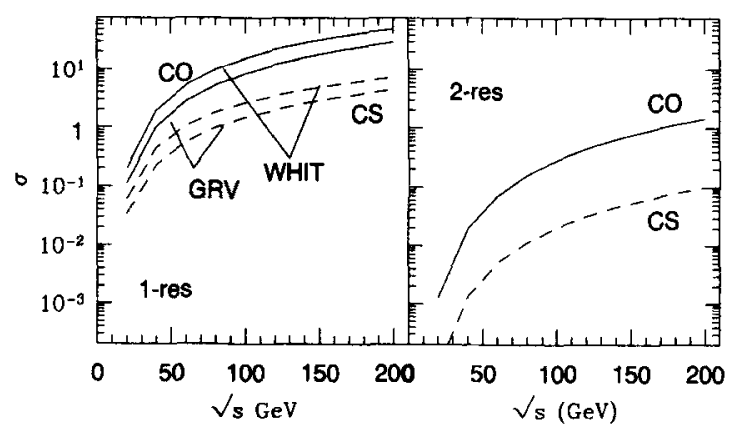

Figure 1. The CO (solid lines) and CS (dashed lines) contributions to the $J / \psi$ production cross section integrated in the range $p_{T}>1 \mathrm{GeV}$ are plotted as a function of $\sqrt{s}$ for 1-res and 2-res processes for GRV [12] parametrizations of the photon densities. The 1-res cross section is also shown for the WHIT parametrization [13] of densities. 


\section{Quantum chromodynamics}

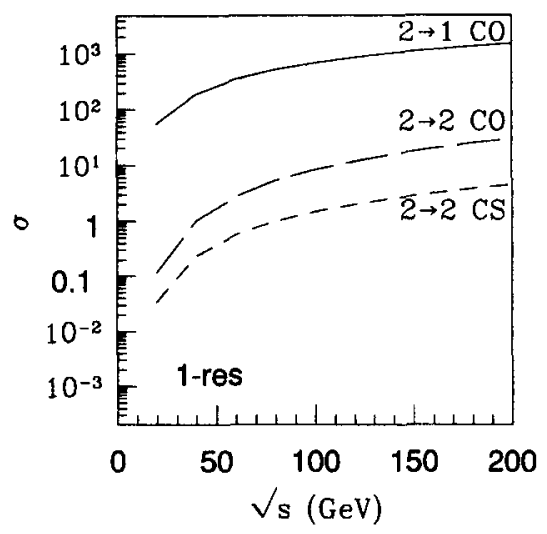

Figure 2. The (purely octet) $J / \psi$ production cross section from $2 \rightarrow 1$ subprocesses is shown as a function of $\sqrt{s}$ for 1-res processes for GRV densities in comparison with the $\mathrm{CO}$ and $\mathrm{CS}$ contributions from the $2 \rightarrow 2$ subprocesses.

Furthermore, even in the case of 1-res $J / \psi$ production, the largest contribution to the total cross section is actually the inelastic scattering at zero $p_{T}$, arising from the $2 \rightarrow 1$ subprocesses $\gamma g \rightarrow J / \psi$, rather than the large- $p_{T}$ cross section coming from the $2 \rightarrow 2$ subprocesses described above. Since the $2 \rightarrow 1$ diagrams only arise from configurations where the $(c \bar{c})$ pair in the final state is in an octet state, this process is likely to furnish an independent estimate of colour octet matrix elements in $J / \psi$ production.

In the case of $e p$ processes, the zero $p_{T} 2 \rightarrow 1$ subprocesses are contaminated by large elastic contributions since both contribute at $z \rightarrow 1$. Hence it is impossible to separate the inelastic contribution from the elastic one. There exists a model of the elastic process based on pomeron exchange [10] where the pomeron is approximated as being a gluon pair. The elastic cross section then behaves as $\left(g_{T}(x)\right)^{2}$ where $x$ is the momentum fraction of the target $T$ (proton) that each gluon carries. For $e^{+} e^{-}$processes, the elastic contribution will measure the pomeron in the photon, and this is expected to be much smaller than the pomeron in the proton. Hence the contamination of the zero $p_{T}$ inelastic events due to the zero $p_{T}$ elastic events is expected to be small. We therefore compute the zero $p_{T}$ (purely $\mathrm{CO}$ ) inelastic contribution to $J / \psi$ production at various colliders; we see, from figure 2 , that these are very much larger than the $2 \rightarrow 2$ contributions. Especially at TRISTAN, where event rates are likely to be low, and events are mostly at small $p_{T}$, this constitutes the dominant contribution. Such a measurement will therefore yield an independent measurement of the octet matrix elements, $n=\left[{ }^{1} S_{0}, 8\right]$ and $\left[{ }^{3} P_{J}, 8\right]$, which dominate this subprocess. Note that we have used the lowest order cross section calculation; the NLO contribution to this process have been computed [11] and, to a good approximation for the integrated cross section, can be incorporated into a $K$-factor multiplying the LO contribution, with $K \sim 1.3$.

To get estimates of event rates at current colliders such as TRISTAN and LEP, we let the $J / \psi$ decay electromagnetically to a lepton pair. We predict about 17 (29) events at TRISTAN (LEP-2) with no cuts on the lepton pair. Realistic acceptance cuts on the lepton angle and $p_{T}$ should reduce the event rates at TRISTAN by approximately a factor two but only by about $10 \%$ in the case of LEP-2. Accurate estimates will be presented in a future work. 


\section{K Sridhar}

In the case of larger $p_{T}$ events, the situation is not so promising, since the production rate falls very rapidly with $p_{T}$. What may be interesting to examine is whether rapidity cuts will enhance the colour octet contribution or else distinguish in some way the $\mathrm{CO}$ from the CS part. We leave this question to future work.

In short, analysis of $J / \psi$ production at $e^{+} e^{-}$colliders such as TRISTAN is already underway. We predict that the largest fraction of the event sample will come from the zero $p_{T} 2 \rightarrow 1$ events which have only a colour octet part. The data will therefore be able to estimate the colour octet matrix elements rather accurately and supplement data from the Tevatron.

Finally, we remark that there is a further uncertainty in $e^{+} e^{-}$collisions compared to $e p$ collisions since the parton densities in the photon are not as well known as those in the proton. Various parametrizations of the parton densities in a photon exist; we show differences due to two of them in figure 1 . While the overall normalization of the cross section does seem to vary with choice of parametrization, it turns out that the $p_{T}$ dependent differential cross section is not sensitive to the choice of parametrization.

\section{References}

[1] CDF Collaboration, F Abe et al, Phys. Rev. Lett. 75, 4358 (1995); 79, 578 (1997)

[2] P Cho and A K Leibovich, Phys. Rev. D53, 6203 (1996)

[3] G T Bodwin, E Braaten and G P Lepage, Phys. Rev. D51, 1125 (1995); erratum: Phys. Rev. D55, 5853 (1997)

[4] H1 Collaboration: S Aid et al, Nucl. Phys. B472, 3 (1996) ZEUS Collaboration: J Breitweg et al, Z. Phys. C75, 215 (1997)

[5] M Cacciari and M Krämer, Phys. Rev. Lett. 76, 4128 (1996)

P Ko, J Lee and H S Song, Phys. Rev. D54, 4312 (1996)

[6] G Japaridze and A Tkabladze, preprint hep-ph/9803447 (1998)

[7] E L Berger and D Jones, Phys. Rev. D23, 1521 (1981)

R Baier and R Rückl, Nucl. Phys. B201, 1 (1982); Z. Phys. C19, 251 (1983)

[8] M Drees and R M Godbole, J. Phys. G21, 1559 (1995)

[9] M Cacciari and M Krämer, in Proceedings of the Workshop on Future Physics at HERA, hep$\mathrm{ph} / 9609500$

[10] M G Ryskin, R G Roberts, A D Martin and E M Levin, Z. Phys. C76, 231 (1997)

[11] A Petrelli, M Cacciari, M Greco, F Maltoni and M L Mangano, hep-ph/9707223 (1997) F Maltoni, M L Mangano and A Petrelli, hep-ph/9708349 (1997)

[12] M Glück, E Reya and A Vogt, Phys. Rev. D46, 1973 (1992)

[13] K Hagiwara, M Tanaka, I Watanabe and T Izubuchi, Phys. Rev. D51, 3197 (1995) 